\title{
Pressure Control of a Nonlinear System with a Linear-PMSM and a Standard Inverter
}

\author{
Christian Junge ${ }^{1}$, Florian Senicar ${ }^{2}$, Ralf Wegener ${ }^{1}$, Member IEEE, Stefan Soter ${ }^{1,2}$, Member IEEE \\ ${ }^{1}$ Electrical Machines and Drives Group, University of Wuppertal, Germany \\ http://www.ema.uni-wuppertal.de \\ ${ }^{2}$ Retostronik GmbH, Gevelsberg, Germany \\ ralf.wegener@ieee.org; stefan.soter@ieee.org
}

\begin{abstract}
This paper deals with a high dynamic hydraulic pressure control. The pressure is generated by an inverter driven linear drive which applies a force on a hydraulic cylinder. The linear drive has to generate a pressure profile into the hydraulic system and has to keep it at a constant value after reaching the demanded pressure. The controlled system, containing hydraulic oil, dissolved air and overall three spring packs, is showing a highly non-linear characteristic. The control cascade of the inverter is based on a standard position control loop, which is enhanced by a PI based pressure controller, a dynamically adapted position feed-forward and an active antiwindup of the controller in order to provide optimal control behavior of the nonlinear system.
\end{abstract}

Keywords - Control, Permanent Magnet Linear Drive, Nonlinear System, PMSM

\section{Motivation}

Hydraulic actuators require a continuously available high oil pressure which is usually generated by a hydraulic pump. The rated power of the pump is considerably high, consuming a lot of energy even when no work is done. If a system additionally requires the availability of multiple possible pressure levels, the system gets very complex. In order to reduce energy consumption and complicity drastically, the standard hydraulic pump is exchanged by a permanent magnet linear synchronous motor which exerts a force to a hydraulic cylinder. This generates superior control possibilities over a standard hydraulic system.

In order to control the generated system pressure, a sophisticated control algorithm is required. This control algorithm needs to be implemented into a standard inverter in order to achieve a competitive pricing of the complete system. This concept allows a great reduction of the energy consumption, better control possibilities for the complete system, as well as new methods of fault diagnosis.

\section{Design of THE LINEAR DRIVE CONCEPT}

This particular tubular linear drive concept consists of three main parts, a stator which is build with discrete coils mounted on poles in radial direction, a permanent magnet armature and an internal position sensor based on the hall-effect. Figure 1 presents a simplification of the real design. It shows one half of a sectional drawing of one basic segment of the linear actuator. The rotation axis is below of the lower edge of the drawing.

The stator comprises a pipe and three stars (one for every phase). Each star is composed of eight winding cores and a ring in the center. For a better saturation of the winding area the star

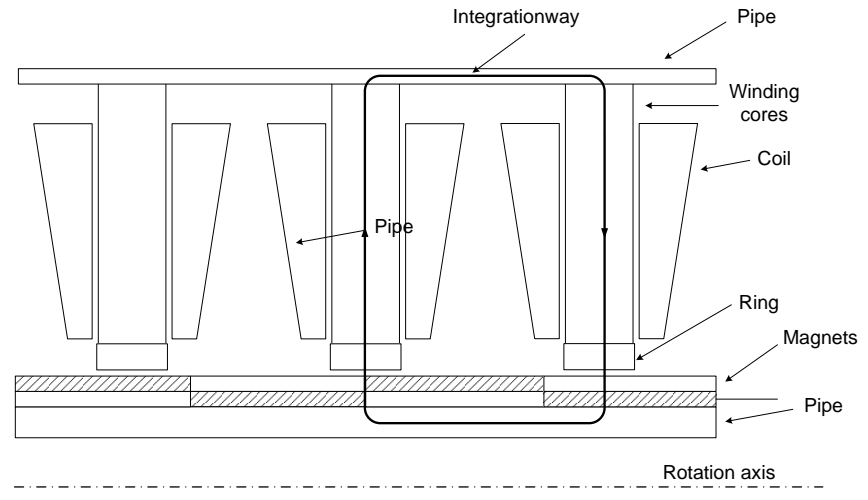

Fig. 1. Sectional drawing of the linear machine

of the second phase is shifted about 22.5 degrees concerning the position of the stars of the first and third phase (figure 2). The arrangement of eight discrete coils per phase offer the possibility of four operating voltages which can be implemented by different types of wiring. The following connections are available: all stator windings can be connected in series or parallel or just four times two windings parallel or two times four windings parallel.

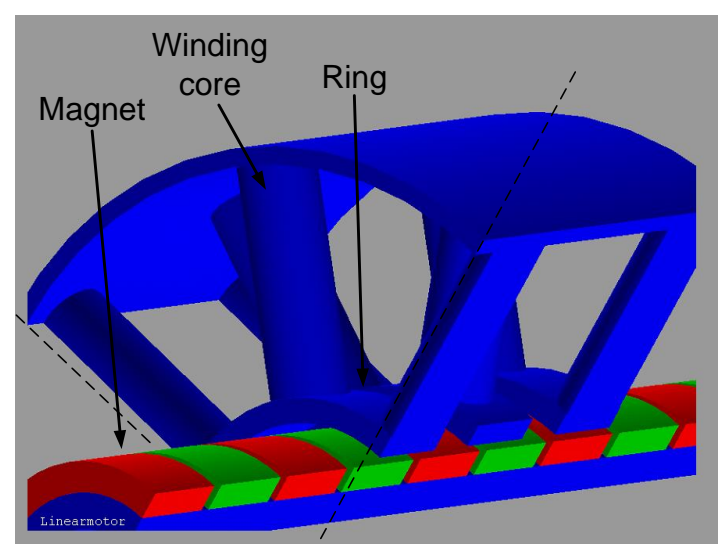

Fig. 2. Configuration of the shifted winding cores

The corresponding armature consists of a steel pipe and four magnets mounted on the surface. For this particular design the radial magnetization was chosen to minimize the magnetic flux distance outside of the ferromagnetic material. Only the small 
airgap and the magnet height must be overcome by the magnet flux as shown in figure 1 . This results in improved efficiency. Additionally the cogging force can be reduced by eliminating the flux density, which is concentrated at the magnet edges caused by the radial flux. The segments may be arranged axially in succession in any desired manner, resulting in modularity with respect to the actuation stroke and/or actuating force.

\section{GENERAL DESCRIPTION OF THE CONTROLLED SYSTEM}

Figure 3 shows the simplified structure of the whole system. The PMSM linear drive generates a force $F_{\text {Mot }}$ onto the hydraulic cylinder A which excites a pressure $P$ into the oil line. Over cylinder $\mathrm{B}$ this pressure is dissipated into a force $F_{\text {Res }}$ which is mounted onto springs. When no hydraulic pressure is applied, the cylinder B has its minimum volume, while the springs have a certain prestress. When applying the maximum allowed pressure, the volume of the cylinder has its maximum.

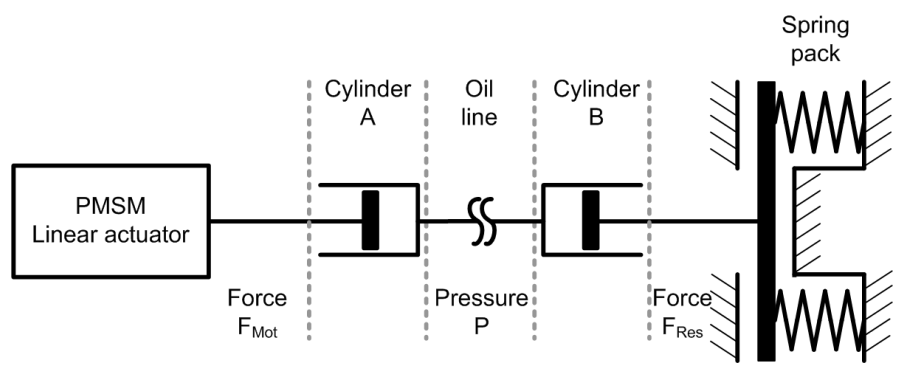

Fig. 3. Structure of the controlled system

Figure 4 shows the characteristic behavior of the specified hydraulic system depending on the position of the linear drive. In the beginning of the curve the pressure is rising up to 20bar while the position of the linear drive is changing about $10 \mathrm{~mm}$. This part of the curve is determined by the compression of the air which is dissolved in the oil. The prestressed springs do not show any movement up to this point, because the generated force of cylinder B is smaller than the force of the springs. Above 20 bar the pressure is not changing significantly while the position is changing around $80 \mathrm{~mm}$. This is caused by the spring characteristic.

$$
\Delta x=\frac{F}{k}
$$

After the springs have been compressed to their maximum point, the characteristic is determined only by oil- and air compression. According to this, the rate of change of the drive position decreases rapidly. A small movement of the linear drive will result in a great change of the oil pressure. Figure 4 shows the high nonlinearity of this system. In the final application the curve of the spring compression in the middle of the shown diagram is not relevant, because there will be no pressure set point for the control loop in this area. In fact only 4 set points are necessary to run the application. P0 is the minimum pressure which is applied always on the system. By starting the application, the linear drive compresses the springs and hold the position on nearly half of the maximum force (P1). After a short while the motor increases the pressure to setpoint $\mathrm{P} 2$ and hold it as long as the actual application is running. By stopping the application the linear drive decreases the pressure to level P3 rapidly and after a short while set point $\mathrm{P} 0$ will be reached by decreasing the pressure once again.

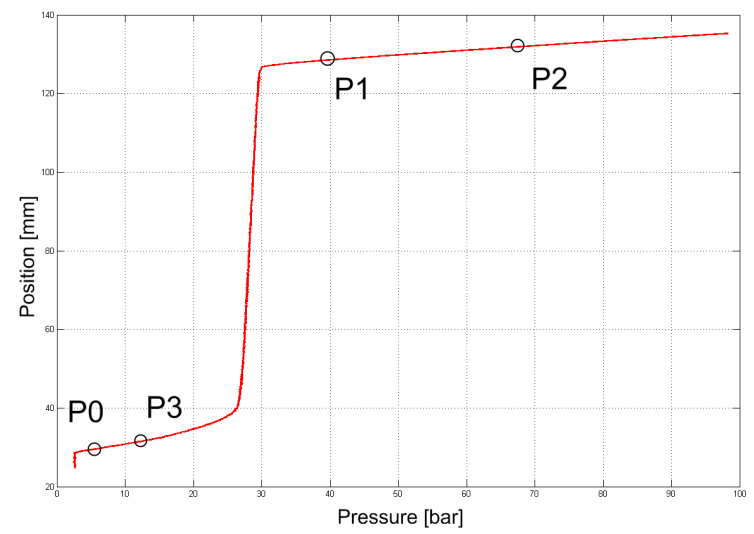

Fig. 4. Resulting pressure of the hydraulic system depending on the position of the linear drive

\section{POSSIBILITY OF FORCE- OR POSITION CONTROL}

The pressure of the hydraulic system is injected by a hydraulic cylinder, which is driven by the force of a linear drive. As an easy approach, a force controlled linear drive can be used to control the pressure of the hydraulic directly. Because of the direct interrelationship between the generated force and the resulting oil pressure, the position and the speed are not necessary to be controlled at all.

$$
P=\frac{F}{A}
$$

Even a measurement of the pressure does not seem to be necessary since with a field oriented control, the generated force is controlled directly. This results in an open loop pressure control. Because of specifications, requesting a high dynamic behavior of the hydraulic, this solution is impracticable:

It is very important, to be able to reduce the pressure from a high value to zero rapidly. Using the open-loop pressure control a pressure set-point of zero will have the result, that no force will be generated at all. The linear drive will be moved backwards due to the existing pressure. It is not possible to reduce the pressure with the drive in an active manner, which heavily decreases system dynamics.

Another possible attempt might be to implement the static pressure plot shown in fig 4 to realize an open loop pressure control based on a position controlled drive. The pressure set point value is equivalent to a position set point which should be reached as fast as possible. This type of control is highly susceptible to variation of system parameters. If the temperature of the oil increases, the pressure characteristic will move as shown in fig. 5. A fast increase of the pressure will result in another disturbing effect. As the dissolved air is being compressed, the temperature as well as the volume increases. After the drives rests at the 


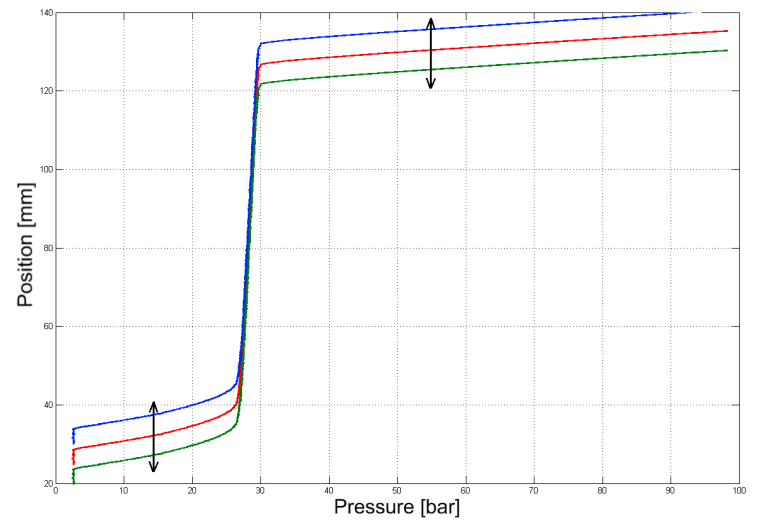

Fig. 5. Temperature variant characteristic of the hydraulic system

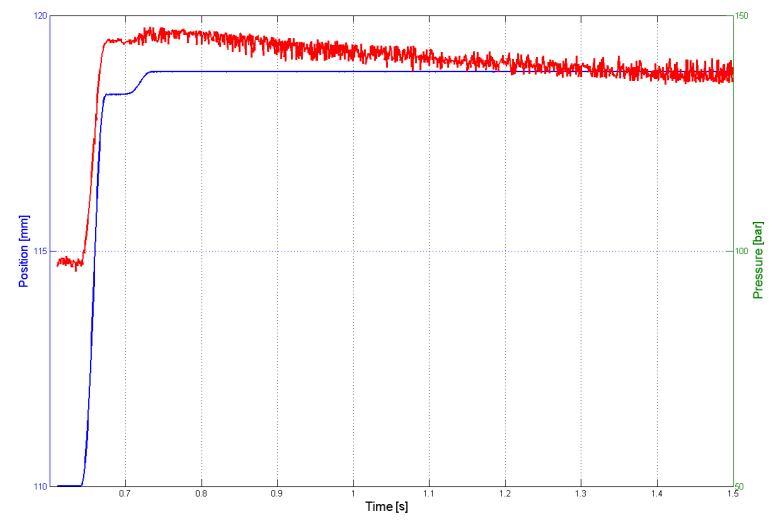

Fig. 6. Decrease of pressure after moving linear drive to constant position

position set point, the air cools down. Because of the resulting decrease of volume, the pressure will also decrease. The drive vary the position in order to keep the pressure on a constant level. Figure 6 shows the characteristic of the pressure after a sudden change of the position. This clearly shows, that an active pressure controller is required to meet the requested specifications.

\section{ReAlized Pressure CONTROL}

To fulfill the required specifications, the following realization is proposed: The control loop is based on a position controlled drive. The set points of the position controller will be determined by an additionally implemented PI-based pressure controller. The integral part of the controller ensures that there will be no permanent offset error.

It has been already mentioned, that the pressure controller has only four different set points. Due to this small amount it is possible to keep independent controller parameters for each of the four set points. This ensures an optimized performance of the controller at every point although the hydraulic system is very nonlinear.

\section{IMPROVING CONTROL DYNAMIC WITH POSITION FEED-FORWARDING}

A position feed forward control is used to increase the speed of the pressure control whenever a change of the set point occurs. The feed forward control is based on a lookup table, which contains a resulting position for every possible set point. This position is added to the actual output of the pressure controller. This feed forward table will move the drive to a position where the pressure is expected to be close to the requested value. The pressure controller itself will merely have to adjust the deviation from that point. The feed forward table improves the response time of the hydraulic system significantly. But however, an inevitable change in temperature or leakage in the hydraulic system will strongly modify the performance of the control loop, since the pressure controller itself is not set up to move the drive that fast. In order to utilize the enhancements of the feed forward table, it is necessary to adapt this table dynamically.

Whenever the linear drive enforces a constant pressure, the actual motor position can be used to update the feed-forward table. At the time, the control logic creates a new set point, the current position will be latched by the inverter and copied back into the table. Figure 7 shows the events where the actual positions will be processed

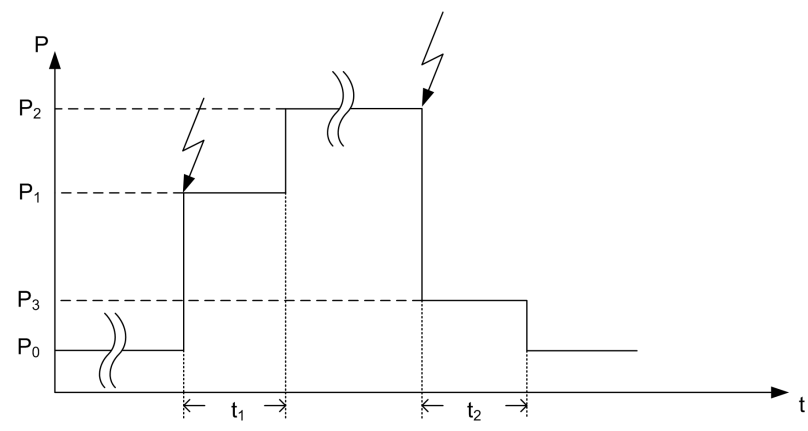

Fig. 7. Example pressure set point diagram over time.

The first shown event displays the appropriate time to store the position value for $\mathrm{P} 0$. The second shown event is used to store the position for the pressure value P2. In order to receive adequate position values for the feed-forward-table, it is necessary to latch the values while the actuator is in a steady state. In general the feed-forward set point table is updated each time a new set point event is generated. The previously pending set point reflects the index of the table that is updated. By employing this method, it can be assured that the controlled linear drive is capable of compensating the expansion of the oil due to a change of temperature.

\section{ACTIVE DAMPING}

Figure 8 shows the large position difference the linear drive will have to overcome in order to follow a sudden change of the set point. The resulting pressure diagram of such a sudden set point change is shown in figure 9.

It is obvious, that during the time the drive moves with its full force (for approx. 50ms), there will be an inevitable difference between the set point and the actual pressure. This will cause an 


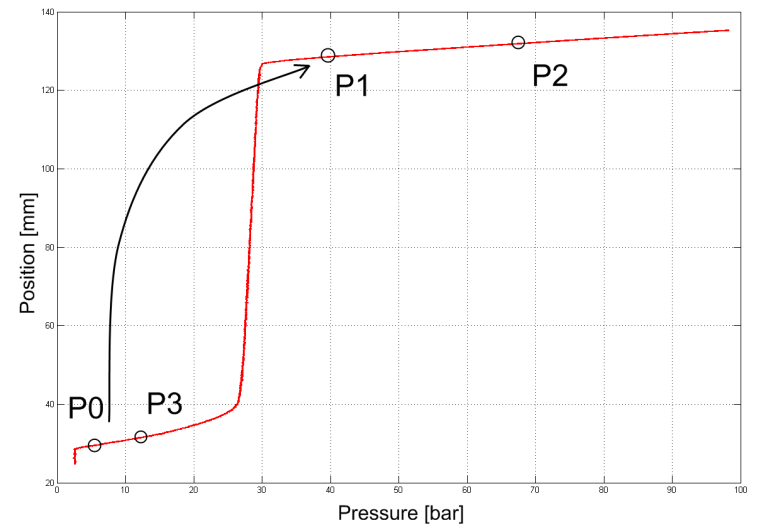

Fig. 8. Applicable set points that can be requested by the control logic

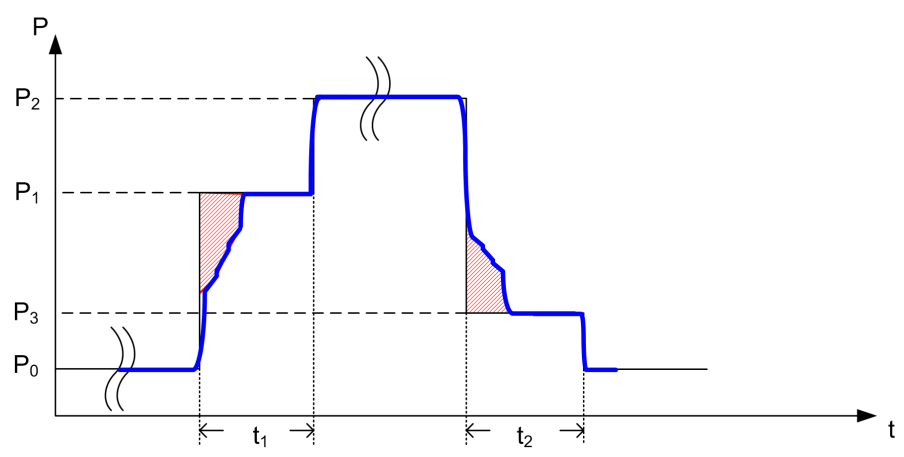

Fig. 9. Example of the pressure-time-diagram

inappropriate wind-up of the integral part of the PI based pressure controller. This will lead to an overshoot of the pressure value which is not allowed by the application and therefore has to be avoided strictly. Since the integral part of the controller is definitely mandatory in order to achieve a good steady state performance, a work-around is needed to compensate this problem. The main idea is, that while the actuator is moving a long distance due to the position-feed-forward table, the integral memory will be continuously reset. The temporary suppression of the integral type controller is necessary for the state changes from P0 to P1 as well as from P2 to P3. The integral controller will be suppressed until the measured actual pressure value is close to the set point value, or whenever the actuator reaches the position set point value from the feed-forward table.

\section{MEASUREMENTS}

Figure 10 shows a measured pressure-time-diagram of the hydraulic system in forward direction. The blue line represents the position while the red line represents the pressure during the filling-phase.

In forward direction the linear drive moves from a pressure of 4 bar and an absolute position of $85 \mathrm{~mm}$ up to $\mathrm{P} 1$ which is at about 85 bar. To reach this pressure the motor has to move around $70 \mathrm{~mm}$. The position is reached after a time of about $70 \mathrm{~ms}$. The next step is after $400 \mathrm{~ms}$, when the linear drive has to push the system up to P2 at 140bar. In this case the position moves only

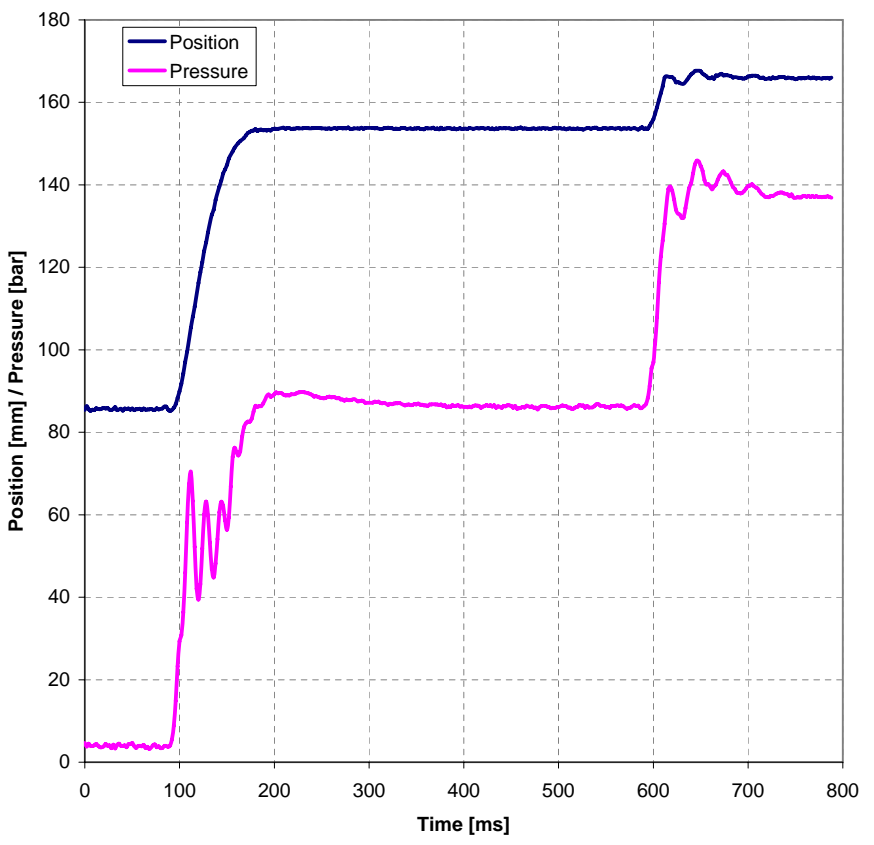

Fig. 10. Measured pressure and position in forward direction

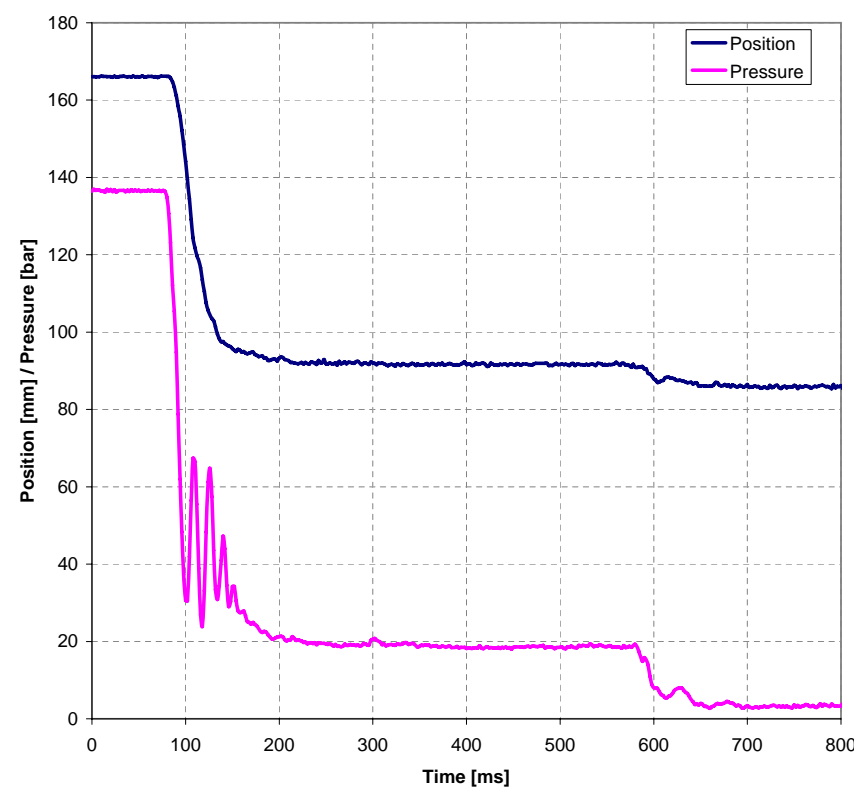

Fig. 11. Measured pressure and position in backward direction

about $8 \mathrm{~mm}$, while the pressure moves from $85 \mathrm{bar}$ up to $140 \mathrm{bar}$. Figure 11 shows a measured pressure-time-diagram of the hydraulic system in backward direction. In this case it is necessary to move from the pressure P2 about 140bar down to P3 nearly 20bar. To reach this pressure, the linear drive has to move $80 \mathrm{~mm}$. This movement is reached in less than $100 \mathrm{~ms}$. To reach the final pressure of about $5 \mathrm{bar}$ the motor has to move $5 \mathrm{~mm}$.

There are some oscillations of the pressure in the middle and end part of the diagrams. They are caused by the air in the system which is more than estimated in the beginning of the development. The next step is to reduce the air in the system by using 
other filling methods.

This high performance control results are achieved with the structure shown in figure 12 by combining all above mentioned control algorithms.

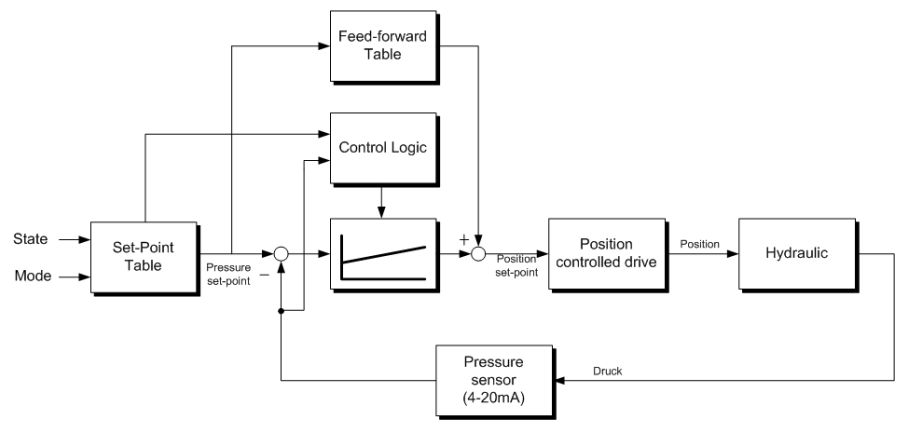

Fig. 12. Resulting pressure control loop

\section{CONCLUSION}

In the paper a specially designed pressure controller based on a standard inverter is figured out in order to drive an highly nonlinear system. The pressure controller has been tested and verified with an external control unit, and later implemented directly into the inverter. The designed control structure is able to fulfil all the requirements that were made concerning dynamics and accuracy.

Due to the implemented pressure controller containing an integral element, there is no permanent offset while providing a constant pressure. With the advanced position feed forward, the pressure controller is assisted during the phases where a new pressure level is requested, resulting in a very fast response time. An overshooting of the actual pressure is suppressed by temporarily deactivating the integral behavior of the pressure controller. The heavily non-linear characteristic of the hydraulics has been taken into account by providing different parameters for the PI based pressure controller for each available set point.

All required logic to run and monitor the hydraulic system has been implemented into a standard inverter. Only two inputs are required to run the hydraulics.

\section{REFERENCES}

[1] Florian Senicar Erweiterung, Optimierung und Test einer StandardRegelkaskade für umrichtergespeiste Linearaktuatorsysteme, Diplomarbeit am Lehrstuhl EAM, Universität Dortmund, März 2007, Chapter: 5 and 6, pages: 35 - 55

[2] William Drury Modeling and High Performance Control of Electric Machines (IEEE Press Series on Power Engineering) John Chiasson Wiley-IEEE Press; 1 edition

[3] Irving M. Electric Motors and Control Techniques Gottlieb McGrawHill/TAB Electronics, 2. edition (February 1, 1994)

[4] William Drury Control Techniques, Drives \& Controls Handbook (Iee Power \& Energy Series, 35) Institution Electrical Engineers, 1st edition (July 15, 2001)

[5] Werner Leonhard Control of Electrical Drives Springer; 3rd ed. edition (September 21, 2001)

[6] Andrzej W. Ordys, Damien Uduehi, Michael A. Johnson Process Control Performance Assessment: From Theory to Implementation (Advances in Industrial Control) Springer; 1 edition (February 16, 2007)

[7] Schröder D. : Elektrische Antriebe - Regelung von Antriebssystemen 2. Auflage. Springer Verlag Berlin 2001 ISBN 3-540-41994-2

[8] Fischer R. : Elektrische Maschinen 7. Auflage. Carl Hanser Verlag München Wien 1989 ISBN 3-446-15600-3
[9] Harald Wertz: Automatisierte Inbetriebnahme der Drehzahlregelung für rotierende Direktantriebe mit hohen Rundlaufanforderungen. Shaker Verlag Aachen 2005 ISBN 3-8322-4007-1

[10] William S. Levine The Control Handbook (Electrical Engineering Handbook) CRC-Press; 1 edition (April 23, 1996)

[11] Richard Valentine Motor Control Electronics Handbook McGraw-Hill Professional; 1 edition (May 1, 1998) 